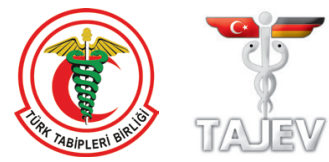

\title{
Pharmacokinetic, pharmacodynamic, and clinical aspects of ovulation induction agents: A review of the literature
}

\author{
Serkan Kahyaoğlu1, Bülent Yılmaz², Ahmet Zeki Işık \\ 1Department of Obstetrics and Gynecology, Zekai Tahir Burak Women's Health Training and Research Hospital, Ankara, Turkey \\ 2Department of Obstetrics and Gynecology, İzmir Katip Çelebi University Faculty of Medicine, Tepecik Training and \\ Research Hospital, IVF Unit, İzmir, Turkey \\ 3Assisted Reproductive Technologies Unit, Medical Park Hospital, İzmir, Turkey
}

\section{Abstract}

Controlled ovarian hyperstimulation is a key step for successful outcomes of assisted reproductive technique cycle outcomes. Many medications are available, which are commonly useed solely or in combination to achieve multiple follicular development. Pharmacokinetic, pharmacodynamic, and clinical information of ovulation induction drugs deserve to be elucidated for every individual patient before commencing infertility treatment. New concepts and new treatment protocols are introduced as ovulation physiology is understood by infertility specialists. Increasing treatment success by minimizing aderse effects is a milestone of all ovarian stimulation protocols that use these novel interventions. Achievement of a satisfactory cycle outcome includes retrieval of sufficient oocytes, a single clinical pregnancy, and avoidance of ovarian hyperstimulation syndrome. In this review, we evaluate the current literature to determine the most reliable and relevant information about the most used ovulation induction drugs. (J Turk Ger Gynecol Assoc 2017; 18: 48-55)

Keywords: Pharmacokinetic, pharmacodynamic, ovulation induction, infertility

Received: 30 May, 2016 Accepted: 10 August, 2016

\section{Introduction}

Pharmacokinetics is the study of drug metabolism in the body according to the rates of three processes: absorption, distribution, and elimination. Pharmacodynamics is the study of the mechanism of action by which drugs exert their pharmacologic effects; the binding of a drug to its target receptor or enzyme followed by a signal transduction pathway by which the receptor activates second messenger molecules, and finally the description of intracellular processes altered by the impact of the drug are components of the pharmacokinetics. Pharmacogenetics and pharmacogenomics are the study of the role of genetic inheritance in individual variation to drug response. Administration of a drug to different individuals can result with different clinical results based on the pharmacogenomic variability among individuals rather than pharmacokinetics. Individualisation of drug therapy can be tailored in the future by using pharmacogenomic information. Recently, infertility became a relatively common public health problem because of the increased prevalance of advanced childbearing age of women. Ovulation induction treatment accompanied by artificial insemination or assisted reproduction are commonly used in infertile women. Pharmacokinetic, pharmacodynamic, and pharmacogenetic aspects of commonly used infertility drugs should be known to improve cycle outcomes. In this review, we aimed to discuss these clinical issues by evaluation of the current published literature regarding ovulation induction agents.

The ovulation induction agents that are commonly used during infertility treatment are shown in the Table 1. 


\section{Antiestrogens (selective estrogen receptor modulators, aromatase inhibitors)}

\section{a. Selective estrogen receptor modulators}

Estrogen reseptor modulators exert partial agonist and antagonist effects according to the tissue estrogen receptor content and estrogen availability level. Selective estrogen receptor modulators (SERMs) act by inhibiting the negative feedback effect of circulating estrogen on the hypothalamic pituitary unit (1). Clomiphene citrate (CC), tamoxifen, and raloxifene are three commonly used SERMs in women's health care.

$\mathrm{CC}$ is well absorbed from the gastrointestinal tract when administered orally. The commonly used daily dosage of CC is between 50-150 mg. Lower than $50 \mathrm{mg}$ doses can be needed for the hyperresponder patient group, especially patients with polycystic ovary syndrome (PCOS). Although rarely needed, higher than $150 \mathrm{mg}$ doses increase the antagonistic effect of $\mathrm{CC}$ on the endometrium and cervix, which is not warranted. $\mathrm{CC}$ is metabolized by hepatic transformation and excreted by feces which increases its bioavailability. High binding capacity to plasma proteins, entering enterohepatic cycle and accumulation in fatty tissues incerases the elimination half life of CC (5 days). Tamoxifen has a slightly higher elimination half life than CC (7 days). CC is exactly a weak estrogen agonist and a moderate estrogen antagonistic molecule. SERMs act on estrogen receptor containing tissues such as the hypothalamus, pituitary, ovary, endometrium, vagina, and cervix by competing with estrogen and decreasing the intracellulary estrogen receptor content. $\mathrm{CC}$ contains two isomeric forms, both of

Table 1. Commonly used ovulation induction agents

Estrogen antagonists
Clomiphene citrate
Letrozole

Insulin sensitizing agents

Metformin

Gonadotropins

- Urinary FSH- recombinant FSH- long acting FSH (corifollitropin alfa)

- Recombinant LH- 1 FSH+ 1 LH (hMG)- 2 FSH+ 1 LH

- Urinary hCG- recombinant (rec) hCG

- Urinary-hMG [1 LH (hCG derived) + 1 FSH]- highly purified (hp) hMG

- Pure FSH-hpFSH

- Urinary hCG- hphCG- rechCG

\section{GnRH analogs}

GnRH agonists

GnRH antagonists

FSH: follicle-stimulating hormone; LH: luteinizing hormone; hMG: human menopausal gonadotropin; hCG: human chorionic gonadotropin; GnRH: gonadotropin-releasing hormone which include different clinical efficacy. Zuclomiphene is the less potent form with long elimination half time, which still exists in the body during early pregnancy achieved by utilization of $\mathrm{CC}$ for ovulation induction. Enclomiphene is the more potent form with short elimination half time which mainly exerts the clinical effect of $\mathrm{CC}$ following oral administration. $\mathrm{CC}$ is a category $\mathrm{X}$ drug but congenital anomaly rates are similar to the normal population. Enclomiphene is the more potent form with a short half life.

In two observational studies, ovulation and pregnancy rates seemed to be improved for patients with PCOS who were treated with tamoxifen following $\mathrm{CC}$ failure $(2,3)$. According to a Cochrane review conducted by Brown et al. (4), pregnancy rate, ovulation rate, miscarriage rate, live birth rate, and ongoing pregancy rate were similar between ovulation induction with $\mathrm{CC}$ and tamoxifen.

\section{b. Aromatase inhibitors}

Anastrazole and letrozole are nonsteroid competetive inhibitors of aromatase. These drugs have been developed for treatment of locally-advanced and metastatic breast cancer of postmenopausal women. Following oral administration, their elimination half time is 2 days. Hot flushes, nausea, headache, vaginal bleeding, and backache are adverse effects. The estrogen suppresion effect of aromatase inhibitors (AI) are dose dependant. The hypothalamo-pituitary-ovarian axis remains intact during ovulation induction treatment and this advantage results with monofollicular ovulation and lower multiple pregnancy rates. Absence of hostile antiestrogenic effect of $\mathrm{CC}$ on endometrium and cervix is another benefit of AIs. In a prospective randomized trial conducted by Diamond et al. (5), ovarian stimulation using letrozole resulted in a significantly lower rate of multiple pregnancy accompanied by a lower rate of live birth when compared with gonadotropin, but not when compared with CC treatment among women with unexplained infertility. Legro et al. (6) conducted another prospective randomized trial and they concluded that when compared with clomiphene, higher live birth and ovulation rates were achieved with ovulation induction using letrozole among infertile women with PCOS. Roque et al. (7) performed a systematic review based on randomized controlled trials comparing cycle outcomes of CC and letrozole among patients with PCOS. A statistically significant increase in the live birth and pregnancy rate was detected in the letrozole group when compared with $\mathrm{CC}$ use [relative risk $(\mathrm{RR})=1.55$ and $\mathrm{RR}=1.38$, respectively]. Ovulation, miscarriage, and multiple pregnancy rates between the two groups were found similar. The authors concluded that regarding live birth and pregnancy rates, ovulation induction using letrozole results with better cycle outcomes when compared with CC in patients with PCOS (7). Letrozole's pharmacodynamic beneficial effects result with 
higher pregnancy rates when compared with CC. Letrozole has shorter elimination half time (45 hours) than CC. Accumulation of $\mathrm{CC}$ within the body results with extended depletion of estrogen receptors accompanied by hostile effects on estrogen sensitive genital tissues. Letrozole increases the biosynthesis of endometrial receptivity markers such as integrins. In 2005, an oral presentation at an American Society of Reproductive Medicine meeting increased concerns regarding congenital malformation and teratogenicity risks of letrozole. This presentation has since been critisized because of the design of the study and the lack of publication in a peer-reviewed journal. Contrarily, cardiac and congenital abnormality rates of pregnancies achieved with $\mathrm{CC}$ have been found increased in some studies $(8,9)$. Tulandi et al. (10) performed a multicenter study comparing the neonatal outcome of 514 letrozole pregnancies with $297 \mathrm{CC}$ pregnancies in 2006 and they concluded that congenital malformation and chromosomal abnormality rates of letrozole and $\mathrm{CC}$ were similar ( $2.4 \%$ vs. $4.8 \%$, respectively). In addition, the cardiac anomaly rate of $\mathrm{CC}$ was significantly higher than letrozole (1.8\% vs. $0.2 \%$, respectively; $\mathrm{p}=0.02$ ).

\section{Metformin}

Metformin is a biguanide oral antidiabetic medication that increases the sensitivity of insulin receptors in peripheral cells. Adding metformin to treatment cycle protocols for increasing pregnancy rates among patients with PCOS is a matter of debate. In a systematic review, Palomba et al. (11) concluded that infertile patients with PCOS treated with gonadotrophins for in vitro fertilization (IVF)/intracytoplasmic sperm injection (ICSI) cycles, implantation rates seemed improved but pregnancy or live birth rates did not increase by using metformin despite lower rates of ovarian hyperstimulation syndrome (OHSS) and miscarriage $(11,12)$. Specific phenotypes and features of patients with PCOS who will benefit from metformin should be defined before liberally advising metformin to all patients with PCOS. Longer than 3 weeks administration of metformin has been found to decrease miscarriage rates [odds ratio (OR) $0.41,95 \%$ confidence interval: $(0.21$ to 0.78$), p=0.0086]$. In a Cochrane database review by Tso et al. (13) in which the clinical effects of metformin treatment before and during IVF or ICSI in women with PCOS were evaluated, the authors concluded that despite significantly beneficial effects for OHSS prevention, no conclusive evidence has been detected for improved live birth rates by using metformin treatment before or during assisted reproductive technique (ART) cycles. Unlike Palomba et al. (11), they emphasized that the use of this insulin-sensitising agent increased clinical pregnancy rates without exerting any beneficial effect on abortus rate, retrieved oocyte number, total gonadotropin dose, stimulation time, fertilization and cycle cancellation rate (13).

\section{Gonadotropins}

Follicle-stimulating hormone (FSH), luteinizing hormone (LH), human chorionic gonadotropin (hCG), and thyroid stimulating hormone are heterodimer glycoprotein hormones including alfa and beta subunits. Alfa subunits of these hormones are made up of same 92 aminoacides. The beta subunit is responsible for the biologic specificity of the hormone. The serum elimination half times of these hormones are relatively short except hCG. Although the beta subunits of LH and hCG have the same rate of $80 \%$, the plasma elimination half time of hCG is 10 times higher than LH. C terminal peptides and sialic acid residues containing 31 amino acids cause this paharmacocinetic difference. The bioavailability of recombinant $\mathrm{FSH}(\mathrm{rFSH})$ and recombinant $\mathrm{LH}(\mathrm{rLH})$ is around $70 \%$ and $60 \%$ following subcutaneous administration, respectively (14-16). No pharmacokinetic interactions occur between $\mathrm{rFSH}$ and $\mathrm{rLH}$ when administered simultaneously. The serum elimination half time of $\mathrm{rFSH}$ and $\mathrm{rLH}$ is around 24 and 10-12 hours, respectively. Steady state plasma levels are achieved after 3-4 days following repeated $\mathrm{rFSH}$ injections. Among patients whose endogenous hypothalamopituitary axis have been suppressed, rFSH alone can efficiently achieve folliculogenesis and also steroidogenesis despite low serum LH levels. Different clinical responses to the same FSH medication doses are caused by FSH receptor polymorphism, also called pharmacogenetics, rather than pharmacokinetic actions of the drug (14-17).

Batch-to-batch inconsistency, foreign proteins, and unpredictable clinical eficiency are major drawbacks of urine-derived gonadotropins. In a prospective randomized multicenter study, Frydman et al. (18) compared rFSH with urinary FSH according to ART cycle outcomes. Achievement of a higher number of oocytes with lower total doses and shorter stimulation times, rFSH was found more potent than urinary FSH. However, the increased oocyte numbers were not reflected by increased pregnancy rates for $\mathrm{rFSH}$ against urinary FSH.

Stimulation of folliculogenesis in the treatment of infertility has been traditionally conducted by using gonadotropins extracted from the urine of postmenopausal women. Urinederived products consist of a mixture of gonadotropins with unpredictable clinical efficiencies and biologically active mediators such as binding proteins, growth factors, and prion proteins. The variation of exact amount of gonadotropins in human menopausal gonadotropin (hMG) preparations results with diverse effects on gonads during ovulation induction. The hCG content and hMG product increases parallel to the increasing purity of the drug to standardise the biologic activity. hCG is secreted by the embryo and placenta and physiologically supports implantation and pregnancy. The receptor binding 
affinity of hCG is 2 times higher than LH. LH has a shorter serum elimination half life than hCG (23 vs. 32-33 hours, respectively). hCG accumulates in the body significantly and causes LH receptor downregulation unlike $\mathrm{LH}$ itself. Six to $8 \mathrm{IU}$ of $\mathrm{LH}$ is biologically equivalent to $1 \mathrm{IU}$ of hCG, which demonstrates the potency of hCG over LH. Controversy has not been resolved as to whether r-hLH or hCG should be used for ART to increase cycle outcomes (19).

In a prospective observational study, Requena et al. (20) compared endocrine profile of oocyte donors stimulated with FSH plus rLH (2/1 in ratio) or hMG. Although retrieved oocyte numbers following treatment with recombinant gonadotropins were higher than urinary gonadotropins (hMG) (16.5 vs. 11.8; $\mathrm{p}=0.049$ ), harvested metaphase II ooocyte numbers were higher by using urinary gonadotropins (hMG) $(71.2 \%$ vs. $80.6 \% ; \mathrm{p}=0.003)$. When serum steroid hormone levels (estradiol, progesterone, testosterone and androstenedione) were evaluated on the day of triggering and cycle day 6 , slightly elevated levels were detected in the recombinant gonadotropins when compared with the urinary gonadotropins. Comparison of intrafollicular levels of steroid hormones were found statistically insignificant between the two protocols and ongoing pregnancy rates were also similar (46.1\% vs. $46.1 \%$ ) (20).

In the Menopur in GnRH Antagonist Cycles with Single Embryo Transfer (MEGASET) trial, Devroey et al. (21) evaluated the safety and efficacy of rFSH and highly purified menotropin (hphMG) for controlled ovarian hyperstimulation in $\mathrm{GnRH}$ antagonist cycles with mandatory single blastocyst transfer. Although higher oocyte numbers were achieved with $\mathrm{rFSH}$ against hMG, similar MII oocyte numbers were harvested. The authors concluded that despite the significant discrepancy in pharmacodynamic effects, highly purified hMG was found have a similar effect as rFSH in GnRH antagonist cycles with mandatory single blastocyst transfer based on clinical pregnancy rates of both fresh and freeze thaw cycle transfers of day 5 embryos (21).

Recently, a gonadotropin preparation that includes $\mathrm{rFSH}$ and rLH 2/1 in ratio was commercially developed. Dosing studies performed on hypogonadotropic hypogonadism patients that evaluted the clinical eficacy of this new drug revealed that 75 IU of LH were sufficient for optimal folliculogenesis (22). Some studies in the literature have demonstrated the nourishing effects on cycle outcomes and ovarian response rates of addition of LH activity to stimulation regimens in certain groups of patients. Women aged older than 35 years, those with diminished ovarian reserve, and women with $\mathrm{LH}$ receptor polymorphisms are theoretical candidates for this approach. Adding $\mathrm{LH}$ to the treatment protocol activates theca cells to produce more androgens, which are eventually converted to estrogens in granulosa cells to increase the estrogenic milieu within the ovarian follicle and also oocyte quality (23).

Pacchiarotti et al. (24) conducted a prospective randomised trial to compare IVF outcomes in ovarian stimulation protocols with recombinant FSH plus recombinant $\mathrm{LH}$ (2/1 in ratio) versus hMG. Treatment with rFSH plus rLH or with hMG was found to produce the same results in terms of implantation rates, pregnancy rates, and embryo quality. Although a statistical difference in oocyte quality, with a better quality in the hMG group was detected, this difference was levelled because of the total number of oocytes retrieved, which was higher in the rFSH plus rLH group, thus the total number of MII oocytes was similar in both groups at the expense of higher OHSS rates for rFSH plus RLH group. The reduction of the amount of FSH used in the hMG group also led to lower cost of the IVF cycle (24).

Bosch (25) published a review article regarding the pharmacologic characteristics and clinical applications of rFSH plus rLH (2/1 in ratio). Although the 2:1 combination of r-hFSH and r-hLH seems to be an optimum ovulation induction regimen regarding safety and clinical efficacy in patients with hypogonatrophic hypogonadism, use of this drug combination in ovarian stimulation for IVF remains controversial because the target population that may receive a benefit from this combination therapy is not well defined. Patients needing $>3000$ IU rFSH during $\mathrm{COH}$, patients showing plateau on follicular growth, and those with inadequate response after 7 days r-FSH have been suggested as candidates for adding $\mathrm{LHH}$ to stimulation regimens based on previous studies (25).

In a systematic review and meta-analysis, Lehert et al. (26) suggested that in poor responders, r-hLH supplementation of r-hFSH compared with rhFSH alone may result in significantly higher oocyte numbers, clinical pregnancy rates, and ongoing pregnancy rates. Based on this entity, Humaidan et al. (27) are currently conducting a randomized controlled multicenter trial to explore the possible advantages of a fixed-dose combination of r-FSH plus r-LH over r-FSH monotherapy in patients with poor ovarian response (POR) according to the definition determined in the European Society of Human Reproduction and Embryology (ESHRE) Bologna criteria.

\section{Long-acting gonadotropins}

Corifollitropin alfa is a long acting recombinant FSH, which acts for 7 days following administration to support folliculogenesis. Although pharmacodynamic actions of long-acting rFSH is the same as with $\mathrm{rFSH}$, the serum elimination half time of longacting $\mathrm{rFSH}$ is 65 hours, which is twice that of $\mathrm{rFSH}$. Dose finding studies revealed that patients weighing above and below 60 $\mathrm{kg}, 100 \mu \mathrm{g}$ and $150 \mu \mathrm{g}$ long-acting $\mathrm{rFSH}$ are recommended for clinical efficiency (28). In a Cochrane database meta-analysis, Pouwer et al. (29) revealed that although the use of a medium 
dose (150 to $180 \mu \mathrm{g}$ ) of long-acting rFSH seemed to be a safe and equally effective treatment option when compared with daily rFSH in women with unexplained subfertility, reduced live birth rate in women receiving a low dose (60 to $120 \mu \mathrm{g}$ ) of longacting rFSH compared with daily rFSH was also observed.

The safety and effectiveness of long-acting FSH for use in hyperor poor responders and in women with all causes of subfertility is an area of current research. In a systematic review and metaanalysis including 4 randomized trials, Mahmoud Youssef et al. (30) concluded that corifollitropin alfa in combination with daily GnRH antagonist seemed to be an alternative for daily rFSH injections in view of efficiency and safety profile among normoresponder patients undergoing controlled ovarian hyperstimulation in IVF/ICSI treatment cycles.

\section{Recombinant human chorionic gonadotropin versus urinary human chorionic gonadotropin}

hCG is used for final maturation of oocytes during ART cycles. Urine-derived hCG has some disadvantages compared with recombinant hCG (rhCG) such as batch-to-batch inconsistency, uncontrolled source, and unpredictable biologic activity. Chang et al. (31) compared the efficacy and safety of $250 \mu \mathrm{g}$ and 500 $\mu \mathrm{g}$ of rhCG with $10000 \mathrm{IU}$ of urinary hCG (uhCG) in ART in a randomized controlled prospective study. As the primary end point of the study, total harvested oocyte numbers were similar for both groups. Based on the results of this study, rhCG was found effective and tolerable in terms of induction of final follicular maturation and luteinization for women undergoing ART procedures. Youssef et al. (32, 33) performed two consecutive Cochrane metaanalyses to assess the safety and efficacy of subcutaneous rhCG and high-dose rLH compared with intramuscular uhCG for inducing final oocyte maturation triggering in IVF and ICSI cycles and they concluded that equivalent pregnancy rates and OHSS incidences were found between rhCG or rhLH and uhCG when used for final follicular maturation in IVF. According to these findings, the authors recommended using uhCG as the best selection for final oocyte maturation triggering in IVF and ICSI treatment cycles.

\section{Gonadotropin-releasing hormone agonist trigger for final oocyte maturation}

hCG has been used as a surrogate for midcycle LH peak to induce final oocte maturation before oocyte retrieval in ART. The relatively long elimination half time of hCG obtains a luteotrophic effect during the luteal phase, but also increases the OHSS risk. Despite obtaining a stimulus for final oocyte maturation, ovulation triggering with hCG has no beneficial effect on endometrial receptivity and oocyte quality when compared with spontaneous ovulation (34). The FSH surge accompanies the LH surge during physiologic ovulation that triggers natural cycles. This midcycle surge of FSH is thought to promote nuclear maturation of the oocyte, cumulus cell accumulation, and $\mathrm{LH}$ receptor formation on granulosa cells. When GnRH antagonists were introduced to the market, the use of $\mathrm{GnRH}$ agonists for final oocyte maturation came into consideration again.

Pioneer studies in this field resulted with disappointment regarding low pregnancy rates and high abortion rates of IVFET cycles triggered with GnRH agonists (35). Modifications of luteal phase support solved this clinical problem and nowadays GnRh agonists are more frequently used for final maturation, especially for patients with increased OHSS risk. Although GnRH agonist trigger strategy seems to decrease OHSS risk with satisfactory pregnancy rates by using modified luteal phase support; early OHSS can still occur even when embryo transfer is deferred (36-38). Oocyte donors, high responser patients, patients who demand fertility preservation, and also normal responder patients are suggested as the target groups for GnRH agonist trigger. During the luteal phase of ART cycles triggered with $\mathrm{GnRH}$ agonists, the relatively shortstanding $\mathrm{LH}$ surge and central inhibition of gonadotropin secretion due to supraphysiological serum estradiol levels causes depletion of LH support, which is needed by the corpora lutea to enhance implantation by secretion of progesterone and also many other implantation favoring mediators. Although luteal estradiol supplementation is not needed for ART cycles triggered with hCG, this intervention is strongly recommended until the $7^{\text {th }}$ gestational week during ART cycles triggered with $\mathrm{GnRH}$ agonists (38). Humaidan et al. (36) suggested administering 1500 IU hCG intramuscularly during oocyte retrieval procedures when GnRH agonists have been used for final oocyte maturation of $\mathrm{GnRH}$ antagonist cycles. This intervention has dramatically lowered abortion rates and boosted the pregnancy rates for this group of patients.

Different doses of different GnRh agonists have been successfully used for final oocyte maturation in the literature. Youssef et al. (39) performed a Cochrane metaanalysis to evaluate the differences between GnRH agonists and HCG in terms of safety and effectiveness for triggering final oocyte maturation in IVF-ICSI among women undergoing a GnRH antagonist protocol. Unlike the Humaidan group, the authors concluded that when GnRH agonists were used for final oocyte maturation in fresh autologous cycles, lower live birth rates, lower ongoing pregnancy rates, and a higher rates of early miscarriage were achieved. Youssef et al. (39) recommended the use of GnRH agonists as an oocyte maturation trigger for women who are spared for fresh transfers, who are oocyte donors, and who demand to freeze autologous oocytes for fertility preservation. Recently, Engmann et al. (40) 
reviewed the advantages and potential drawbacks of GnRH agonist triggering by performing a strengths, weaknesses, opportunities and threats (SWOT) analysis. Based on this analysis modality, the authors recommended intensive luteal support with transdermal oestradiol and intramuscular progesterone alone if peak serum oestradiol is 4000 or more $\mathrm{pg} / \mathrm{mL}$ after GnRHa triggering or dual triggering with $\mathrm{GnRH}$ agonist and hCG 1000 IU if peak serum oestradiol is less than $4000 \mathrm{pg} / \mathrm{mL}$. The recommendations of the same group based on the follicle number were as follows: administration of hCG 1500 IU $35 \mathrm{~h}$ after GnRH agonist trigger if there are less than 25 follicles $\geq 11 \mathrm{~mm}$ on the day of ovulation trigger, or freeze all oocytes or embryos if there are over 25 follicles (40).

\section{Gonadotropin-releasing hormone antagonist (short) versus gonadotropin-releasing hormone agonist (long) protocol}

Al-Inany et al. (41) conducted a Cochrane metaanalysis for comparing these mostly used $\mathrm{COH}$ protocols. They investigated the safety and effectiveness of $\mathrm{GnRH}$ antagonists by comparison with the long protocol of GnRH agonists for ovarian stimulation in ART cycles. In this review, the authors concluded that when compared with long GnRH agonist protocols, the antagonist protocol was associated with a wide decrease in OHSS rates and similar live birth rates $(41,42)$. The same group recently conducted a Cochrane systematic review and similar live birth rates were observed between $\mathrm{GnRH}$ antagonist and long $\mathrm{GnRH}$ agonist protocols. When compared with GnRH agonists, GnRH antagonist-based protocols lowered the incidence of all OHSS severity grades (OR 0.61). The miscarriage rates were found similar between these two protocols. The cycle cancellation rate following POR to ovulation induction was higher in women who received $\mathrm{GnRH}$ antagonist protocols compared with $\mathrm{GnRH}$ agonist protocols (OR 1.32) (43). Based on these results, GnRH antagonist protocols seem to be the best and safest protocol for patients with high baseline OHSS risk. Contrarily, GnRH agonist protocols result with higher oocyte yield than $\mathrm{GnRH}$ antagonist protocols among poor responder patients. Sunkara et al. (44) performed a randomized controlled study among poor responders undergoing IVF treatment. The number of oocytes retrieved was significantly higher with long GnRH agonists compared with short agonist regimens $(4.42 \pm 3.06$ vs. $2.71 \pm 1.60)$ and similar between long agonist and antagonist regimens (4.42 \pm 3.06 vs. $3.30 \pm 2.91)$. Total gonadotropin dose and duration of stimulation were significantly higher using long agonist regimens compared with short agonist and antagonist regimens. The ongoing pregnancy rates were $16.2 \%$ with antagonist protocols and $8.1 \%$ with long and short agonist protocols $(\mathrm{p}=0.48)$. Based on these results, the authors concluded that long GnRH agonist and antagonist regimens can be a better selection as ovulation induction regimens for poor responders, whereas the short agonist regimen seems to be a less effective treatment strategy because fewer oocytes are retrieved (44). Al Inany et al. conducted a Cochrane systematic review including 73 RCTs, with 12212 participants, comparing GnRH antagonist to long-course GnRH agonist protocols. Although the quality of the selected studies for this systematic review was moderate, the use of $\mathrm{GnRH}$ antagonist was found associated with a substantial reduction in OHSS without reducing the likelihood of achieving live birth when compared with long-course GnRH agonist protocols (43).

In conclusion, rational use and administration of ovulation induction drugs necessitate evaluation of pharmacokinetic, pharmacodynamic, and clinical aspects of each individual medication based on pharmacologic and clinical evidence. This clinical practice will eventually increase the success of ovulation induction protocols performed for infertility treatment and decrease the health threatening risks that arise from the treatment burden.

Ethics Committee Approval: N/A.

Informed Consent: N/A

Peer-review: Externally peer-reviewed.

Author Contributions: Concept - A.Z.I., S.K., B.Y.; Design - S.K.; Supervision - A.Z.I.; Materials - S.K., B.Y.; Data Collection and/ or Processing - S.K.; Analysis and/or Interpretation - A.Z.I., S.K., B.Y.; Literature Review - S.K., B.Y.; Writer - A.Z.I., S.K., B.Y.; Critical Review - A.Z.I., S.K., B.Y.

Conflict of Interest: No conflict of interest is declared by the authors.

Financial Disclosure: The authors declared that this study received no financial support.

\section{References}

1. Brenner GM, Stevens C. Pharmacokinetics. Pharmacology; Chapter 2: 925: 2012.

2. Gulekli B, Ozaksit G, Turhan NO, Senoz S, Oral H, Gokmen O. Tamoxifen: an alternative approach in clomiphene resistant polycystic ovarian syndrome patients. J Pak Med Assoc 1993; 43: 89-90.

3. Dhaliwal LK, Suri V, Gupta KR, Sahdev S. Tamoxifen: An alternative to clomiphene in women with polycystic ovary syndrome. J Hum Reprod Sci 2011; 4: 76-9.

4. Brown J, Farquhar C, Beck J, Boothroyd C, Hughes E. Clomiphene and anti-oestrogens for ovulation induction in PCOS. Cochrane Database Syst Rev 2009: CD002249.

5. Diamond MP, Legro RS, Coutifaris C, Alvero R, Robinson RD, Casson $\mathrm{P}$, et al. Letrozole, Gonadotropin, or Clomiphene for Unexplained Infertility. N Engl J Med 2015; 373: 1230-40.

6. Legro RS, Brzyski RG, Diamond MP, Coutifaris C, Schlaff WD, Casson $\mathrm{P}$, et al. Letrozole versus clomiphene for infertility in the polycystic ovary syndrome. N Engl J Med 2014; 371: 11929. 
7. Roque M, Tostes AC, Valle M, Sampaio M, Geber S. Letrozole versus clomiphene citrate in polycystic ovary syndrome: systematic review and meta-analysis. Gynecol Endocrinol 2015; 31: 917-21.

8. Reefhuis J, Honein MA, Schieve LA, Rasmussen SA; National Birth Defects Prevention Study. Use of clomiphene citrate and birth defects, National birth defects prevention study, 1997-2005. Hum Reprod 2011; 26: 451-7.

9. Davies MJ, Moore VM, Willson KJ, Van Essen P, Priest K, Scott H, et al. Reproductive technologies and the risk of birth defects. N Engl J Med 2012; 366: 1803-13.

10. Tulandi T, Martin J, Al-Fadhli R, Kabli N, Forman R, Hitkari J, et al. Congenital malformations among 911 newborns conceived after infertility treatment with letrozole or clomiphene citrate. Fertil Steril 2006; 85: 1761-5.

11. Palomba S, Falbo A, La Sala GB. Effects of metformin in women with polycystic ovary syndrome treated with gonadotrophins for in vitro fertilisation and intracytoplasmic sperm injection cycles: a systematic review and meta-analysis of randomised controlled trials. BJOG 2013; 120: 267-76.

12. Uysal S, Zeki Isik A, Eris S, Yigit S, Yalcin Y, Ozun Ozbay P. Correlation of endometrial glycodelin expression and pregnancy outcome in cases with polycystic ovary syndrome treated with clomiphene citrate plus metformin: a controlled study. Obstet Gynecol Int 2015; 2015: 278591.

13. Tso LO, Costello MF, Albuquerque LE, Andriolo RB, Macedo CR. Metformin treatment before and during IVF or ICSI in women with polycystic ovary syndrome. Cochrane Database Syst Rev 2014: CD006105.

14. Porchet HC, Le Cotonnec JY, Loumaye E. Clinical pharmacology of recombinant human follicle-stimulating hormone. III. Pharmacokinetic-pharmacodynamic modeling after repeated subcutaneous administration. Fertil Steril 1994; 61: 687-95.

15. le Cotonnec JY, Porchet HC, Beltrami V, Munafo A. Clinical pharmacology of recombinant human luteinizing hormone: Part I. Pharmacokinetics after intravenous administration to healthy female volunteers and comparison with urinary human luteinizing hormone. Fertil Steril 1998; 69: 189-94.

16. Le Cotonnec JY, Loumaye E, Porchet HC, Beltrami V, Munafo A. Pharmacokinetic and pharmacodynamic interactions between recombinant human luteinizing hormone and recombinant human follicle-stimulating hormone. Fertil Steril 1998; 69: 201-9.

17. Yilmaz B, Kelekci S, Savan K, Oral H, Mollamahmutoglu L. Addition of human chorionic gonadotropin to clomiphene citrate ovulation induction therapy does not improve pregnancy outcomes and luteal function. Fertil Steril 2006; 85: 783-6.

18. Frydman R, Howles CM, Truong F. A double-blind, randomized study to compare recombinant human follicle stimulating hormone (FSH; Gonal-F) with highly purified urinary FSH (Metrodin) HP) in women undergoing assisted reproductive techniques including intracytoplasmic sperm injection. The French Multicentre Trialists Hum Reprod 2000; 15: 520-5.

19. Ezcurra D, Humaidan P. A review of luteinising hormone and human chorionic gonadotropin when used in assisted reproductive technology. Reprod Biol Endocrinol 2014; 12: 95.

20. Requena A, Cruz M, Ruiz FJ, García-Velasco JA. Endocrine profile following stimulation with recombinant follicle stimulating hormone and luteinizing hormone versus highly purified human menopausal gonadotropin. Reprod Biol Endocrinol 2014; 12: 10.

21. Devroey P, Pellicer A, Nyboe Andersen A, Arce JC; Menopur in GnRH Antagonist Cycles with Single Embryo Transfer Trial Group. A randomized assessor-blind trial comparing highly purified hMG and recombinant FSH in a GnRH antagonist cycle with compulsory single-blastocyst transfer. Fertil Steril 2012; 97: 561-71.

22. http://www.emea.europa. eu/humandocs/PDFs/EPAR/pergoveris/ H-714-PI-en.pdf
23. Bosch E, Pau E, Albert C. Impact of LH administration on oocyte quality and embryo morphology. Fertil Steril 2006;86: (Suppl 2): S425.

24. Pacchiarotti A, Sbracia M, Frega A, Selman H, Rinaldi L, Pacchiarotti A. Urinary hMG (Meropur) versus recombinant FSH plus recombinant LH (Pergoveris) in IVF: a multicenter, prospective, randomized controlled trial. Fertil Steril 2010; 94: 2467-9.

25. Bosch E. Recombinant human follicular stimulating hormone and recombinant human luteinizing hormone in a 2:1 ratio combination. Pharmacological characteristics and clinical applications. Expert Opin Biol Ther 2010; 10: 1001-9.

26. Lehert P, Kolibianakis EM, Venetis CA, Schertz J, Saunders H, Arriagada $\mathrm{P}$, et al. Recombinant human follicle-stimulating hormone (r-hFSH) plus recombinant luteinizing hormone versus r-hFSH alone for ovarian stimulation during assisted reproductive technology: systematic review and meta-analysis. Reprod Biol Endocrinol 2014; $12: 17$.

27. Humaidan P, Schertz J, Fischer R. Efficacy and Safety of Pergoveris in Assisted Reproductive Technology--ESPART: rationale and design of a randomised controlled trial in poor ovarian responders undergoing IVF/ICSI treatment. BMJ Open 2015; 5: e008297.

28. Fauser BC, Alper MM, Ledger W, Schoolcraft WB, Zandvliet A, Mannaerts BM, et al. Pharmacokinetics and follicular dynamics of corifollitropin alfa versus recombinant FSH during ovarian stimulation for IVF. Reprod Biomed Online 2010; 21: 593-601.

29. Pouwer AW, Farquhar C, Kremer JA. Long-acting FSH versus daily FSH for women undergoing assisted reproduction. Cochrane Database Syst Rev 2015: CD009577.

30. Mahmoud Youssef MA, van Wely M, Aboulfoutouh I, El-Khyat W, van der Veen $\mathrm{F}$, Al-Inany $\mathrm{H}$. Is there a place for corifollitropin alfa in IVF/ICSI cycles? A systematic review and meta-analysis. Fertil Steril 2012; 97: 876-85.

31. Chang P, Kenley S, Burns T, Denton G, Currie K, DeVane G, et al. Recombinant human chorionic gonadotropin (rhCG) in assisted reproductive technology: results of a clinical trial comparing two doses of rhCG (Ovidrel) to urinary hCG (Profasi) for induction of final follicular maturation in in vitro fertilization-embryo transfer. Fertil Steril 2001; 76: 67-74.

32. Youssef MA, Al-Inany HG, Aboulghar M, Mansour R, Abou-Setta AM. Recombinant versus urinary human chorionic gonadotrophin for final oocyte maturation triggering in IVF and ICSI cycles. Cochrane Database Syst Rev 2011: CD003719.

33. Youssef MA, Abou-Setta AM, Lam WS. Recombinant versus urinary human chorionic gonadotrophin for final oocyte maturation triggering in IVF and ICSI cycles. Cochrane Database Syst Rev 2016: CD003719.

34. George K, Kamath MS, Nair R, Tharyan P. Ovulation triggers in anovulatory women undergoing ovulation induction. Cochrane Database Syst Rev 2014: CD006900.

35. Kolibianakis EM, Schultze-Mosgau A, Schroer A, van Steirteghem A, Devroey P, Diedrich K, et al. A lower ongoing pregnancy rate can be expected when $\mathrm{GnRH}$ agonist is used for triggering final oocyte maturation instead of HCG in patients undergoing IVF with GnRH antagonists. Hum Reprod 2005; 20: 2887-92.

36. Humaidan P, Kol S, Papanikolaou EG; Copenhagen GnRH Agonist Triggering Workshop Group. GnRH agonist for triggering of final oocyte maturation: time for a change of practice? Hum Reprod Update 2011; 17: 510-24.

37. Gülekli B, Göde F, Sertkaya Z, Isık AZ. Gonadotropin-releasing hormone agonist triggering is effective, even at a low dose, for final oocyte maturation in ART cycles: Case series. J Turk Ger Gynecol Assoc 2015; 16: 35-40. 
38. Gurbuz AS, Gode F, Ozcimen N, Isik AZ. Gonadotrophin-releasing hormone agonist trigger and freeze-all strategy does not prevent severe ovarian hyperstimulation syndrome: a report of three cases. Reprod Biomed Online 2014; 29: 541-4.

39. Youssef MA, Van der Veen F, Al-Inany HG, Mochtar MH, Griesinger G, Nagi Mohesen M, et al. Gonadotropin-releasing hormone agonist versus HCG for oocyte triggering in antagonist-assisted reproductive technology. Cochrane Database Syst Rev 2014: CD008046.

40. Engmann L, Benadiva C, Humaidan P. GnRH agonist trigger for the induction of oocyte maturation in GnRH antagonist IVF cycles: a SWOT analysis. Reprod Biomed Online 2016; 32: 274-85.

41. Al-Inany HG, Youssef MA, Aboulghar M, Broekmans F, Sterrenburg M, Smit J, et al. Gonadotrophin-releasing hormone antagonists for assisted reproductive technology. Cochrane Database Syst Rev 2011: CD001750.
42. Cicek MN, Kahyaoglu I, Kahyaoglu S. The comparison of microdose flare-up and multiple dose antagonist protocols based on hCG day estradiol (E2), progesterone (P) and P/E2 ratio among poor responder patients in ICSI-ET cycles. Eur Rev Med Pharmacol Sci 2015; 19: 539-44.

43. Al-Inany HG, Youssef MA, Ayeleke RO, Brown J, Lam WS, Broekmans FJ. Gonadotrophin-releasing hormone antagonists for assisted reproductive technology. Cochrane Database Syst Rev 2016: CD001750.

44. Sunkara SK, Coomarasamy A, Faris R, Braude P, Khalaf Y. Long gonadotropin-releasing hormone agonist versus short agonist versus antagonist regimens in poor responders undergoing in vitro fertilization: a randomized controlled trial. Fertil Steril 2014; 101: 14753. 